\title{
RESEARCH ON KEY TECHNOLOGY OF HIGH VOLTAGE LIVE OPERATION BASED ON DOUBLE MANIPULATOR
}

\author{
Jing Guo ${ }^{1}$, Tao Xie ${ }^{1}$, Kai Wei ${ }^{1}$, Xiaoyu Chen ${ }^{1}$, Jianmin Wang ${ }^{1}$ \\ ${ }^{1}$ Maintenance Branch of State Grid Shanxi Electric Power Company, \\ Taiyuan, Shanxi 030006, China \\ Email: gf59731@163.com
}

\begin{abstract}
In recent years, GIS equipment has been widely used in high-voltage power transformation because of its advantages such as high reliability in operation, small footprint, convenient maintenance and so on. However, GIS equipment will be disturbed by the defects of insulating materials and poor installation technology, which will inhibit the normal use of GIS equipment and increase the operation risk of GIS equipment. If there is a failure problem, it will lead to the occurrence of power failure accident, causing economic losses to enterprises and society, and bringing inconvenience to life. In order to reduce the occurrence of such problems, this paper takes GIS equipment circuit breaker fittings as an example to carry out assembly operations with the help of dual-arm high-voltage live working robot, so as to ensure the standardization and effectiveness of GIS equipment circuit breaker fittings assembly operations. At the same time, the path planning of the dual-arm live working robot is carried out under different constraints, and the simulation experiment is carried out. The final simulation results show that the path planning under the unconstrained condition has high safety and feasibility.
\end{abstract}

Keywords: GIS equipment, Circuit breaker, Hardware, Dual-arm robot, Live working.

\section{Introduction}

In the circuit breaker in GIS equipment hardware assembly and split, visual positioning technology is one of the key, to overcome uncertain complex background environment, illumination conditions, dual arm robot weak rigidity and strong electromagnetic interference problems such as interference, can effectively function the positioning of the circuit breaker in GIS equipment hardware, work lay the foundation for subsequent hardware assembly and split ${ }^{[1-4]}$. In addition, there may also be problems such as visual positioning error, robot main structure deformation and dual-manipulator calibration error, which lead to the failure to effectively complete the hole shaft centering operation. Therefore, a set of control strategies need to be designed to control them and assist the assembly and hole searching of circuit breaker fittings in GIS equipment. Aiming at the above problems, this paper will take the assembly of circuit breaker hardware in GIS equipment as an example to analyze the key technologies of the doublemanipulator high-voltage live working robot, and check the path planning of the double-manipulator high-voltage live working robot under different conditions through simulation experiments.

\section{Analysis of Equipotential Operation Process of Double Manipulator System}

The dual-arm system designed in this paper is mainly composed of a gripping arm (H1) and a tightening arm (H2), which is mainly responsible for the disassembly and installation of circuit breaker hardware in GIS equipment. Its mechanistic model is shown in Figure 1.

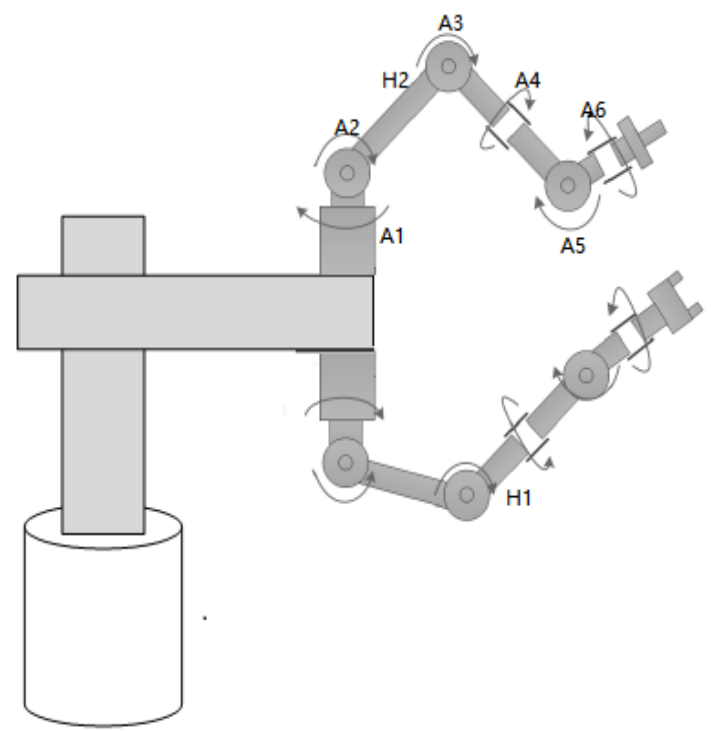

Figure 1: Composition of the two manipulator arms 
In Figure 1, A1, A2, and A3 determine the terminal position, and $\mathrm{A} 4, \mathrm{~A} 5$, and $\mathrm{A} 6$ form a wrist joint orthogonal to each other to determine the terminal posture. Taking the disassembly of circuit breaker hardware in GIS equipment as an example, the equipotential operation process of the dualmechanical ratio system can be subdivided into four parts: First, the visual positioning system is used to determine the location of circuit breaker hardware in GIS equipment, and then the clamping manipulator is moved to a safe area near the hardware to avoid induction discharge problems. Second, tighten the mechanical arm and insert the bolt into it. At this time, the operation platform of the mechanical arm and the circuit breaker hardware in GIS equipment are in the state of equal potential, indicating that the induction arc discharge is over. Third, the use of clamping manipulator clamping hardware, and then use tighten the screw bolt in diagonal way, mechanical arm all bolts loosen after evacuation tight mechanical arm, in the clamping manipulator with hardware, hardware equipotential clamp, etc., so tight tight grab of mechanical arm not clamp and the induced potential difference. Fourth, pull out the L - shaped tool with the supporting mechanical arm, but not more than the safe distance.

\section{Single Visual Positioning Technology of High Voltage Live Working Robot based on Double Manipulator Arms}

\subsection{Visual System Framework Design}

In order to achieve accurate positioning of highvoltage substation GIS equipment, the vision system of dual-manipulator high-voltage live working robot is designed and studied here. Since the object of this paper is the circuit breaker in the GIS equipment in the substation, the design and research will be carried out from the aspect of monocular stereo vision positioning technology. Typically, a singleview camera is placed at the end of the manipulator to meet the operational and positioning requirements in complex environments. The vision system designed in this paper has calibration, measurement and positioning functions, among which, the internal and external parameters of the camera can be obtained by using calibration functions (camera calibration, hand-eye relationship calibration, and the relative position and posture calibration of dual-arm robots), and the real-time control of abnormal parameters can be carried out. At the same time, the transformation relationship between the coordinate system of the single camera and the tool coordinate system of the robot and the posture calibration of the robot can also be obtained. The measurement and positioning function is composed of image processing and coordinate transformation. By using this function, the pictures taken can be processed (including pre-processing, image segmentation, contour extraction, etc.), and then the relative position and posture of the dualarm robot can be obtained by the calibration function. The specific design of the monocular stereo vision positioning system is shown in Figure 2.

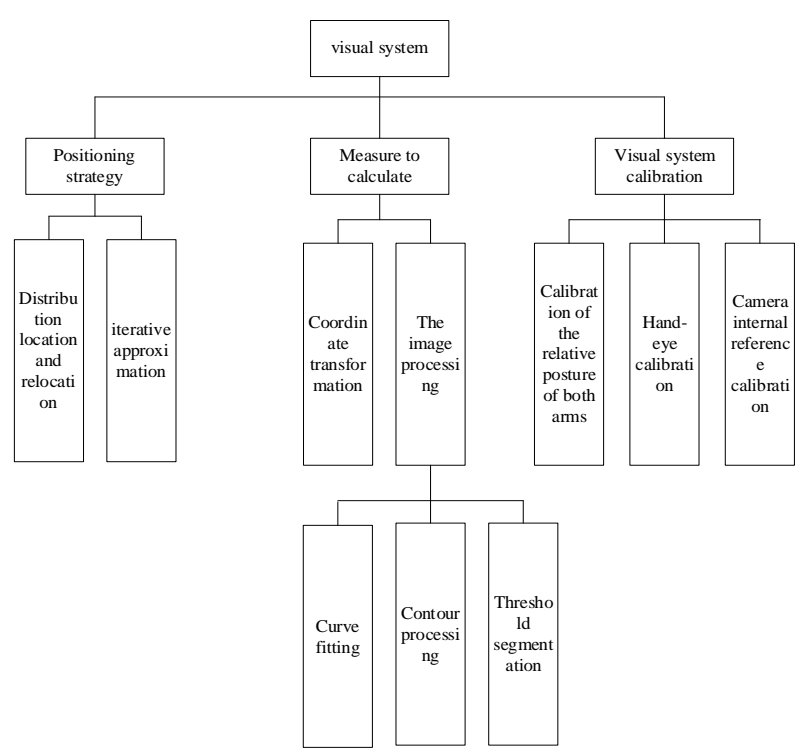

Figure 2: Architecture of monocular stereo vision positioning system

\subsection{Monocular Vision of Weakly Rigid High- Voltage Live Working Robot}

In practice, although object features are constructed by means of points, straight lines and curves, the two positioning schemes of points and straight lines have multi-solution problems, which are inconsistent with the main purpose of this paper. Therefore, the most concise circular marking method is adopted to achieve accurate positioning of weakly rigid high-voltage live working robot. In order to avoid the situation that the position and pose of the wire clamp cannot be captured, three circular features (Mark points) are set here to calibrate the wire clamp, and the three center of the circle are connected to complete the construction of the metal coordinate system ${ }^{[5-6]}$. In the process of image preprocessing, the automatic threshold image method can be used to segment the image to get rid of the influence of illumination factors. The specific segmentation process is shown in Figure 3.

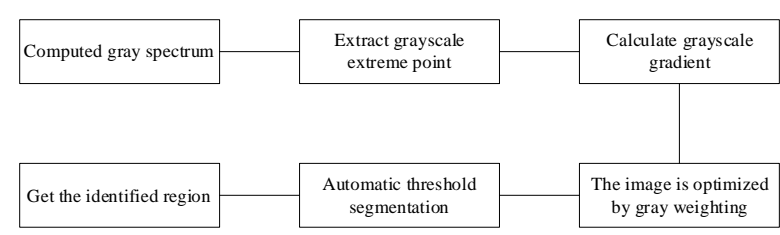

Figure 3: Automatic threshold segmentation process

It can be seen that during image segmentation, the extreme gray point of the image is obtained with the help of gray spectrum, and then the background area is removed, and the marker area is divided into 
several (n) subregions. In all sub-regions, the gray scale $\widetilde{x}_{i}$ corresponding to the intermediate point can be used to represent its approximate gray value. In combination with the corresponding weight of $f_{i}$ as $w_{i}$, Formula 1 can be obtained.

$$
w_{i}=f_{i} / \sum_{k=1}^{n} f_{k}
$$

Using Equation 1, the expected value formula of gray balance can be obtained, as shown in Equation 2.

$$
\widetilde{\mathrm{x}}=\widetilde{\mathrm{x}_{1}} \mathrm{w}_{1}+\widetilde{\mathrm{x}_{2}} \mathrm{w}_{2}+\cdots+\widetilde{\mathrm{x}_{\mathrm{n}}} \mathrm{w}_{\mathrm{n}}
$$

As can be seen from Equation 2, the gray update value corresponding to each sub-region is $\left(\widetilde{x_{1}}-x\right)$. Due to the high balance during gray optimization, the adaptability of dynamic extreme value segmentation to light conditions can be improved, and the image segmentation and extraction can be realized. In fact, environmental interference and surface quality degradation will be encountered in the process of extracting the outline of the Mark point, which leads to the failure to extract the corresponding Mark point well. In order to solve this problem, this paper uses the sub-pixel fuzzy smoothing method to process the edge of the Mark point, so that the edge area is smooth. Although the positioning accuracy is reduced, it can still meet the design requirements, so as to achieve the contour extraction of the Mark point.

\subsection{Monocular Vision Iterative Positioning Method of Weakly Rigid Robot}

When the double robotic arms patrol the circuit breakers in the substation GIS equipment, the movement of the robot and the lift and fall of the robotic arms will affect the visual system's shooting of the circuit breakers in the GIS equipment, resulting in the shooting position error.

Monocular vision positioning technology is sensitive to the shooting Angle and position, which can be understood through the following two aspects: First, in the imaging process, different shooting angles will lead to changes in the image of the edge contour of Mark points, and meanwhile, the edge quality of Mark points themselves will also affect the imaging quality and calculation accuracy. Second, in the image processing, if the shooting Angle and position are different, then the extracted sub-pixel edges will also have certain differences, and the final ellipse characteristic parameters will be different. In order to solve this problem, the iterative approximation method is chosen as the iterative localization algorithm of rigid robot.

\subsection{Positioning strategy for vision system of high-voltage charged robot}

Due to the special working environment of the double-arm live operation robot, the positioning strategy of the monocular vision system needs to be improved continuously to ensure the normal live operation of the double-arm live operation robot. In the research on the positioning strategy of the vision system of the high-voltage charged robot, the system positioning strategy flow is shown in Fig. 4 after comprehensive consideration of the weak stiffness of the metal tool support, the weak rigidity of the platform support and the induced arc discharge problem.

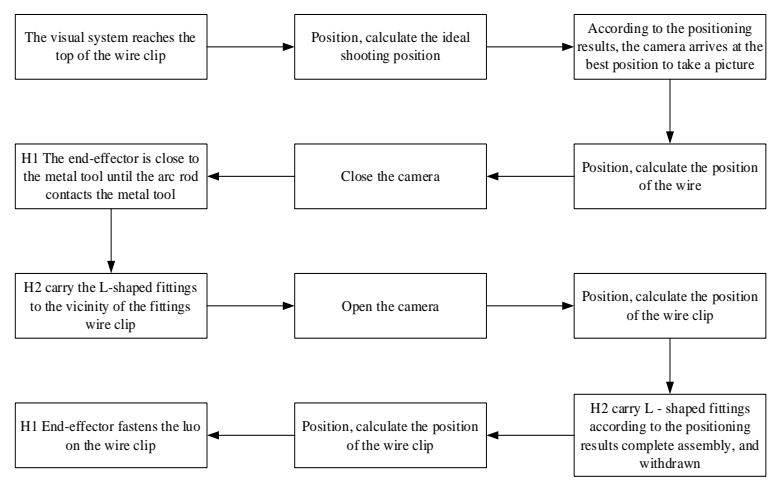

Figure 4: System positioning strategy process

\section{Active Compliance Control Based Wire Assembly of Hardware}

\subsection{Geometric Force Analysis of the Contact Surface of the Shaft Hole of the Metal Tool}

When the circuit breaker in the substation GIS equipment is connected and assembled with the fittings, the fittings wire clamp shall be fixed on the insulating porcelain bottle on the outgoing line of the GIS bus, and then the L-shaped fittings shall be clamped with the help of $\mathrm{H} 1$ to move towards the fittings and complete the assembly. During assembly, circuit breakers and fittings will be interfered by hand-eye relationship calibration error, visual positioning error, and dual-manipulator coordinate system calibration error, etc., resulting in position deviation $C$ and Angle deviation $\theta$ of the line clamp hole axis and L-shaped fittings axis, which leads to hole search failure or clamping and other phenomena. In order to avoid this kind of problem, it is necessary to control the dual-arm robot. Before assembly, the tension $\mathrm{F}_{\mathrm{ACSR}}$ caused by the dead weight of the soft wire, the dead weight of the Lshaped metal tool $G_{L}$ and the dead weight of the mechanical gripper $G_{C}$ have been applied together to form the pre-load, and the force principle is shown in Figure 5 . 


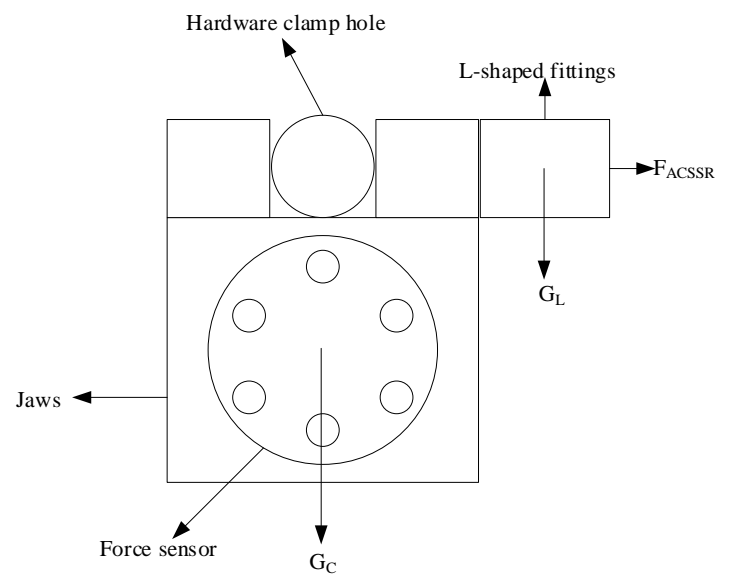

Figure 5: Principle of force

Through force analysis, it can be known that under this tangential load, if the elastic element is used to bend the mechanical gripper, the buffer device is bound to absorb and store the energy of the pre-load first, resulting in the failure of elastic deformation in the direction of the pre-load in the assembly process, and the loss of passive compliant function, so active compliant assembly is required. A force sensor is installed between the end of the mechanical arm and the clamping claw to detect the force of the L-shaped tool in contact with the clamping wire of the tool, and then the mechanical arm is scheduled to be tightened to make corresponding actions for compensation to complete the final assembly. The specific assembly process is shown in Figure 6.

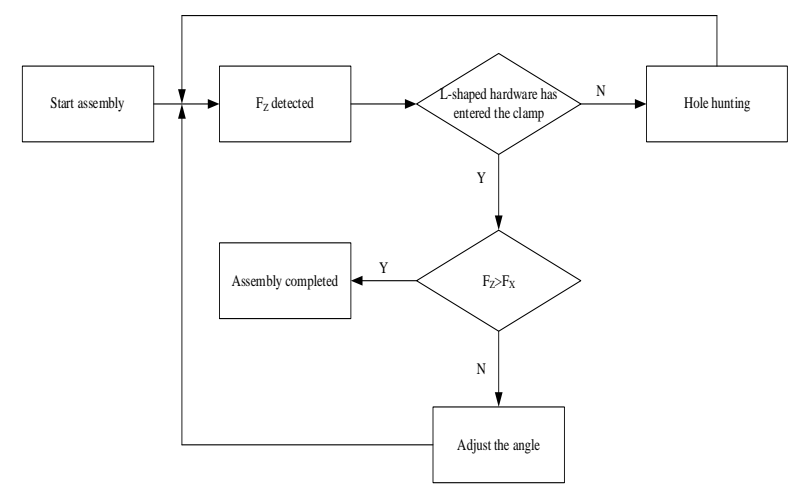

Figure 6: Hardware assembly process

\subsection{Hole Search Process of Tool Shaft}

In this paper, the shaft is tilted into the hole in the hole of the tool shaft searching, and the relative position of the shaft hole is approximated iteratively according to the feedback information of the force sensor. In this way, the interference caused by the complex environment can be avoided, and the hole searching efficiency can be improved. Tool shaft hole search can be divided into three stages: stage one refers to the close; Phase two refers to alignment; Stage three refers to insertion, as shown in Figure 7.

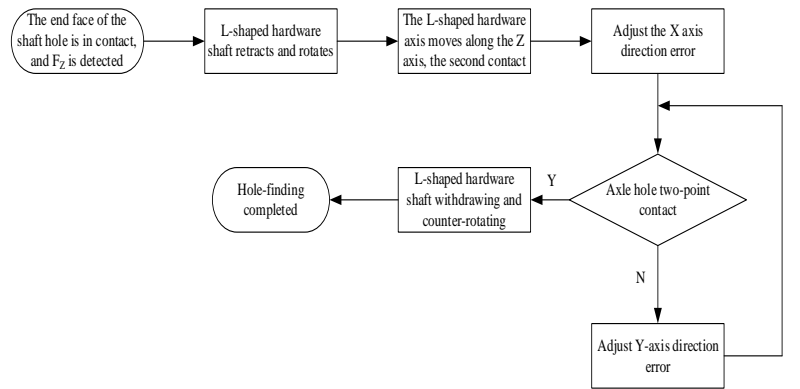

Figure 7: Hole tracing process for fittings

\subsection{Inspection of Fittings Assembly Strategy}

In order to verify the effectiveness of the circuit breaker hardware assembly strategy in GIS equipment, an equal-scale simulated environmental test was carried out. The experimental results are as follows: it took 135s from the first contact of the two parts of the shaft hole to the end of assembly. The results confirm the effectiveness of the proposed tool assembly strategy, which can deal with the problems of large initial contact error of shaft hole and poor robot rigidity, and complete the two-part assembly task of shaft hole of tool.

\section{Path Planning for high voltage live Operation of Dual-Arm Robot}

\subsection{Comprehensive Evaluation Function Analysis}

When the dual-arm robot carries out high voltage live operation, the movement modes of the robot arm end are mainly point-to-point motion (PTP), circular motion (CIRC) and linear motion (LIN). However, attention should be paid to the safety problems and joint overlimit problems in the path planning process of dual-arm live operation. Therefore, path planning here is not a single objective programming problem, but a multiobjective programming problem. In view of the above considerations, the safety margin evaluation function, joint limit approach function and comprehensive evaluation function were established to solve the path planning problem of high-voltage live operation of dual-arm robot $[9,10]$. Among them, the safety margin evaluation function is constructed as follows:

$$
H_{s}=\left\{\begin{array}{cl}
K_{p}\left(d-D_{i s_{\text {safe }}}\right)^{2} & d \leq \text { Dis safe }_{\text {safe }} . \\
0 & d>\text { Dis safe }_{\text {saf }}
\end{array}\right.
$$

In Equation 3, the minimum safe distance between the manipulator and the dangerous boundary is denoted by d; Safety margin evaluation function is expressed by $H_{s}$; The proportion coefficient of the evaluation function is expressed by $K_{p}$. The safety distance threshold is represented by dis. 
When dis greater than the safety distance threshold, there is no need to adjust the path planning for the live operation of the manipulator. When $d \leq$ Dis $_{\text {safe, }}$, the value of $H S$ is inversely proportional to $\mathrm{d}$ and will increase as it decreases. When $\mathrm{d}>$ Dis $_{\text {safe }}$ the evaluation function value is 0 , which means that the live operation of the manipulator can achieve the corresponding safety index. When $H_{s}>S_{P}$ Dis $s_{\text {safe }}{ }^{2}$, it means that the live operation of the manipulator arm does not meet the safety requirements. If these variables are unified into $[0,1]$, then the safety margin evaluation function can evolve into:

$$
H_{S}=\left\{\begin{array}{cl}
\left(\frac{d-\text { Dis }_{\text {safe }}}{d_{D}}\right)^{2} & d \leq \text { Dis }_{\text {safe }} \\
0 & d>\text { Dis }_{\text {safe }}
\end{array}\right.
$$

In order to avoid the dangerous area of the end of the double manipulator arm in live operation robot, the occurrence of joint over-limit problem is avoided. The rotation Angle (a3) of the robotic arm joint A3 should be taken into account, and the midpoint position of the moving region of a3 should be regarded as the gravitational source. Then, the limit approach function $H_{L}$ of the joint should be constructed by means of the artificial potential field method:

$$
H_{L}=S_{L}\left(\alpha_{3}-\frac{\alpha_{3 R}+\alpha_{3 L}}{2}\right)^{2} \quad \alpha_{3} \in\left[\alpha_{3 L}, \alpha_{3 R}\right]
$$

In Equation 5, the proportionality coefficient is expressed by $S_{L}$; The left and right limits of $\alpha_{3}$ are represented by a3L and $\alpha_{3 R}$, respectively. If $\alpha_{3}=\frac{a_{3 R}+\alpha_{3 L}}{2}$, the rotation Angle of the manipulator joint is at the most ideal position, and the function takes A minimum value. When $H_{L}>K_{L}\left(\frac{a_{3 R^{-}} a_{3 L}}{2}\right)^{2}$, the rotation Angle of the robotic arm joint will exceed the motion limit, and there will be movement risk. The joint limit approach function obtained after deviation normalization can be expressed by Equation 6.

$H_{L}=\frac{4}{\left(\alpha_{B R}-\alpha_{B L}\right)^{2}}\left(\alpha_{3}-\frac{\alpha_{3 R}+\alpha_{3 L}}{2}\right)^{2} \quad \alpha_{3} \in\left[\alpha_{3 L}, \alpha_{3 R}\right]$

Equations 4 and 6 are processed by weighted grouping method to form a comprehensive evaluation function, as shown in Equation 7.

$$
\mathrm{H}=\omega_{\mathrm{L}} \mathrm{H}_{\mathrm{L}}+\omega_{\mathrm{S}} \mathrm{H}_{\mathrm{S}}
$$

In Equation 7, the weighting coefficient is represented by $\omega_{i}$, and $\omega_{L}+\omega_{S}=1$.

\subsection{Path planning for high voltage live operation of dual-arm robot}

The path planning of dual-arm robots can be divided into two kinds: the first is the path planning without path constraints, that is, when the manipulator moves from the initial position to the Lshaped tool, there is a target position constraint, but there is no path constraint; The second is path planning with path constraints, that is, there are target position constraints and path constraints when the manipulator starts moving from L-shaped hardware. Among them, the first path planning process is as follows: firstly, using the positioning results of the vision system and the size of the substation structure, the danger boundary information in the base coordinate system of the manipulator and the detection of the target pose are calculated, and then the dynamic space of the selfjoint structure is solved. Then, by using the hand mapping relationship, the key postures in 3D space are projected in the joint structure space to obtain the target configuration geometry. Secondly, safety and joint motion range were taken as constraint conditions, and the comprehensive evaluation function was used to select the optimal target configuration. Finally, path search is carried out in the configuration space to complete the final path planning. The specific process is shown in Figure 8.

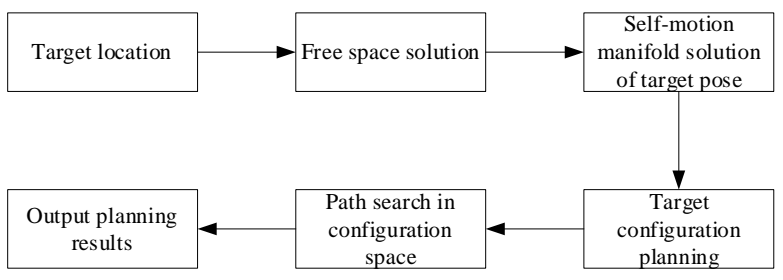

Figure 8: Path planning process without path constraints

The second path planning method is as follows: First, the motion path of the end of the manipulator is processed by the discrete decomposition method, and a set of key postures $T_{i}, i=1,2,3 \cdots, n$, and the motion from $T_{i}$ to $T_{i+1}$ is regarded as self-motion popular motion in the configuration space. Secondly, the end of the manipulator is always operated in the TI pose, and then the configuration of the manipulator is self-motion adjusted to improve its safety and avoid joint problems. Finally, on the premise of not changing the redundant degree of freedom of the manipulator, the manipulator passes through the other joints and moves to the $T_{i+1}$ pose. In conclusion, the comprehensive evaluation function can be expressed by Equation 9 .

$$
\mathrm{H}=\mathrm{H}\left(\mathrm{G}\left(\mathrm{d}_{0}, \mathrm{~T}_{\mathrm{i}}\right)\right)
$$


In Equation 9, the redundant degrees of freedom are represented by $d_{0}$, and the joint pose at the end of the manipulator is represented by $T_{i}$. The selfmotion adjustment process of $T_{i}$ point can be regarded as the process of redundant DOF joints moving towards the direction of $\mathrm{H}$ decreasing. Therefore, an iterative equation of variable-length redundant degrees of freedom can be constructed, as shown in Equation 10.

$$
d_{0}^{\prime}=d_{0}-H \times S_{p} \times \operatorname{sign}\left(\left.H^{r}\right|_{d_{0}}\right)
$$

In Equation 10, the basic step size is represented by $S_{p}$, and the first partial derivative of $d_{0}$ is represented by $\left.H^{r}\right|_{d_{0}}$ in the Ti pose. Therefore, in the presence of path constraints, a path that can not only meet operational safety standards but also avoid joint overrun problems can be selected.

\subsection{Simulation Analysis}

In order to verify the feasibility of path planning and design for live operation of dual-arm robot in this paper, the disassembly operation of circuit breakers in GIS equipment is taken as an example to conduct A simulation experiment. The relevant parameters are set as follows: let $d_{B}=2000 \mathrm{~mm}, \omega_{L}=0.3, \omega_{s}=0.7$, safety distance threshold $d_{D}=400 \mathrm{~mm}$. The initial configuration is $[0,0,-2.58,9.28,0,83.3,0]^{T}$, and H1 moves PTP from the initial position $P_{0}(1100,0,400,180,0,0)$ to the preparing position $P_{1}(1500,400,300,180,0,0)$, then moves upward in a straight line to hold the gold tool $P_{2}(1500,600,0,180,0,0)$, and finally moves in a straight line to pull out the gold tool $P_{4}(1500,600,500,180,0,0)$, so as to realize the live disassembly operation of the circuit breaker gold tool in GIS equipment by the double manipulator.

According to the path planning process without path constraints, the final result is shown in Figure 9.

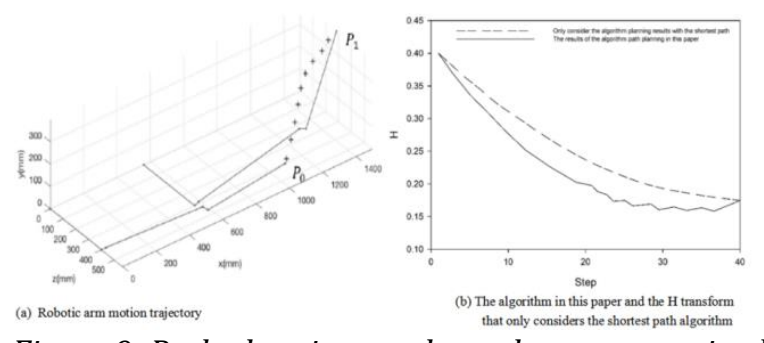

Figure 9: Path planning results under unconstrained conditions

After the dual-manipulator path planning without path constraints is finished, the simulation experiment of dual-manipulator path planning with path constraints is carried out. According to the path planning process, the motion paths between
$P_{1}, P_{2}, P_{3}, P_{4}$, are obtained, as shown in Figure 10 (a). Meanwhile, the relationship between the distance $\mathrm{d}$ between the manipulator and the dangerous boundary plane and joint Angle $\theta_{3}$ is obtained, as shown in Figure 10 (b).

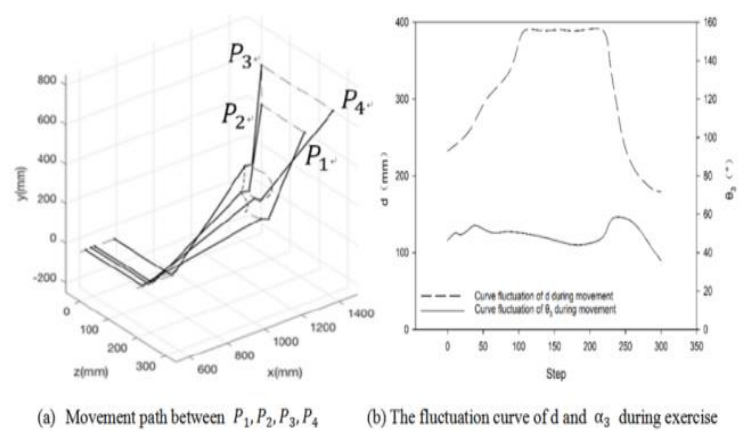

Figure 10: Path planning results with path constraints

In summary, the minimum motion trajectory threshold of the dual-arm live working robot is $170 \mathrm{~mm}$, which belongs to the safe motion range. At the same time, Angle $\theta_{3}$ fluctuates within the range of 30-80, which is lower than the limit value of Angle, and there will be no over-limit problem. Therefore, the feasibility and security of path planning algorithm for live operation of dual-arm robot with path constraints are confirmed.

\section{Conclusions}

To sum up, this paper first analyzes the operation process of the dual-arm robot at the same point, and gives the specific operation process. Secondly, the monocular vision positioning system of the dual-arm high voltage live working robot is designed, the iterative positioning method and positioning strategy of monocular vision are given, and the equal-scale simulation environment test is carried out.

The results confirm the feasibility of the positioning strategy in this paper. Finally, the double arm live working robot path planning, and through the simulation experiments under different conditions of the path planning of feasibility is verified, the results confirmed a dual arm robot under the path constraint between the safety and feasibility of path planning, to ensure that the GIS equipment breaker hardware precision disassembly and assembly work.

\section{Acknowledgement}

This study is supported by State Grid Shanxi Electric Power Company Science and Technology Project: Research and application of key technologies for intelligent detection of suspended dense GIS Equipment Group in UHV Substation (project fund No.: 52051020008A). 


\section{References}

[1] Xu W. "Application Research of GIS High Voltage Combination Appliance in Intelligent Substation," Electronic World, 2020, (14):78-79.

[2] Li L. "Analysis on Operation and Maintenance of GIS Equipment," Digital Communication World, 2020, (08):116-117.

[3] Gan XH. "Fault Diagnosis and Maintenance Analysis of GIS Equipment in Substation," Electronic Technology, 2021, 50(01):142-143.

[4] Wang H, Ma Q, Wei JR, Liu C, Wu X, Feng L, Mi XF. "Experimental Study on Poroity of Insulating Tie Rings for $550 \mathrm{kV}$ GIS Circuit Breaker," High Voltage Appliances, 2021, 57(01):55-61.

[5] Wang YS, Song JH, He P, Zhang Y. "Research on Mark Point Image Repair Based on Mumford \& Shah Algorithm," Journal of Hubei University (Natural Science Edition), 2021, 43(03):271$275+281$.
[6] Bao XM, Wu XR, Lv WT. "Mark Point Location Method of PCB Board Based on IBBS-SIFT Algorithm," Journal of Zhejiang Sci-Tech University (Natural Science Edition), 2019, 41(03):360-366.

[7] Liu CZ, Han XL, Li JC. "Easymptotic Iterative Approximation Method for Cubic Uniform Bspline Expansion Curves," Journal of ComputerAided Design \& Computer Graphics, 2019, (6):899-910.

[8] Liu ZY. "Two-step Evolutionary Iterative Approximation Method and Convergence," Journal of Shaoyang University (Natural Science Edition), 2019, (6).

[9] Li WP, Shen HB. "Research on Linear Discriminant Analysis Algorithm Based on Proximity Function," Electronic Technology, 2017, 46(02):5-7.

[10] Shi L, Lin HM. "A Discussion on Questioning the Comprehensive Evaluation Function of Principal Component Analysis," Mathematics in Practice and Theory, 2020, 50(14):312-320. 